\title{
Recombinant Nematode Anticoagulant Protein c2
}

National Cancer Institute

\section{Source}

National Cancer Institute. Recombinant Nematode Anticoagulant Protein c2. NCI

Thesaurus. Code C69129.

An 85-amino acid recombinant peptide derived from protein c2 of the hemophagocytic hookworm Ancylostoma caninum (a common canine parasite) with anticoagulant activity. Recombinant nematode anticoagulant protein c2 (rNAPc2) binds to circulating activated factor X (FXa) or zymog en factor X (FX) to form a binary complex which subsequently binds to and inhibits membrane-bound activated factor VII/tissue factor complex (FVIIa/T F). When administered prophylactically, this agent may reduce the incidence of deep venous thrombosis without hemostatic compromise. Because rNAPc2 inhibits the formation of the FVIIa/TF protease complex, which may play a role in the cellular signaling of both metastatic and angiogenic processes, it may impede tumor progression. 